\title{
Combining Discriminant Analysis and Neural Networks for Detection of Internal Defects in Mangoes using X-Ray Imaging Technique
}

\author{
Vani Ashok
}

\begin{abstract}
In today's competitive world, quality is considered as the key factor in the modern food industry and the quality of agricultural produce is of main concern for export. Specifically, quality of fruits is of major concern in the export and import industry as it has to conform to the quality norms of the corresponding country. In recent years, non-invasive imaging techniques such as Magnetic resonance imaging (MRI), X-ray, Computed tomography (CT), Nuclear magnetic resonance (NMR), Near infrared (NIR), Ultrasound and Hyper-spectral imaging are being employed to determine the quality of fruits. The "king of fruits", Mango (Magnifera indica Linn) is the most economically important agricultural crop. India being the major producer of mangoes $(50 \%$ of global production)and contributing majority of mango cultivars to the world market needs economical, non-destructive methods for quality evaluation of mangoes. There is a need to develop a nondestructive system that objectively classifies the internal quality of mangoes in real time. In this paper, an X-ray based computer vision methodology is proposed to automatically detect internal defects of mangoes and classify the quality into two groups, "Defective" and "Non-defective". In the proposed methodology we built a dataset of $572 X$-ray images of mangoes and validated it using Discriminant Function Analysis (DFA) predictive model which determines the group membership of each sample in the dataset based on the huge feature space extracted from the sample images. The features that best predicts the group membership were given as inputs to Multilayer PerceptronNeural Network (MLP NN) with scaled conjugate gradient optimization algorithm and the optimized MLP architecture with maximum classification accuracy was determined. The proposed model was able to classify the X-ray image samples into Defective and Non-defective groups with an accuracy of $91.3 \%$.
\end{abstract}

Keywords-X-ray imaging, Non-destructive, Internal Defects, Discriminant Function, Scaled Conjugate Gradient, Neural Networks

\section{INTRODUCTION}

The term quality often describes the degree of excellence of a product and there is an increasing emphasis on the quality of agricultural products, specifically on fruits and vegetables from the consumer end.This has motivated the researchers and food industry across the globe to develop techniques that can be used to assess the quality of the agroproducts.
Revised Manuscript Received on December 12, 2019

Vani Ashok ${ }^{*}$,Computer Science and Engineering, Sri Jayachamarajendra College of Engineering, Mysuru, Karnataka, India.E-mail:vanisj@ sjce.ac.in
There is increased demand for the non-destructive methods for evaluating the quality so that the product is not damaged or destroyed. The quality of fruits is determined by many factors like shape, size, color, weight, texture, firmness, etc. Consumers consider these parameters for determining the quality of the fruit if and only if the fruit is free from internal and external defects. From past five decades lot of efforts has gone into the development of automated, consistent and rapid computer vision techniques to perform identification and classification of external defects of agricultural products. Numerous attempts are also being made to identify the internal defects of fruits but majority of them being destructive in nature. Researchers have managed to find some success in evaluating the internal quality of fruits using various imaging techniques with different spectrums such as MRI, X-ray, CT, NMR, ultrasound, optical, sonic, and NIR radiation [1]. These techniques require combination of effective sensors to measure the physical/chemical properties and computer vision with state-of-the art classification algorithms to establish relationship between measured quality parameters of the product [2]. Among these imaging techniques, recently X-ray, which is predominantly used in medical field, is gaining popularity in food quality evaluation as it is much more convenient, cost effective (when compared to MRI), gives information about the whole fruit and also easily accessible, as it is available in airport and hospitals [3]. Soft X-rays have low penetration power and are able to expose the internal density changes more accurately [4]. Due to these reasons they are more suitable to detect major internal defects of fruits such as presence of insects, cavity/pits, water core, insect damage, fungal infection seed germination and bruise.

Based on two-dimensional (2-D) X-ray imaging technique, neural network has been employed to identify internal damage in apples due to watercore. The classifier was able to categorize the apples into three classes, clean, mild and severe based on different watercore levels using eight extracted features from X-ray images of apples with less than $10 \%$ false positive and negative ratios [5]. $\mathrm{P}$. Chenet. al. have reviewed the quality assessment of fruits and vegetables based on physical properties such as density to detect frost damage in citrus, insect damage in fruits, cavities in potatoes and firmness to separate damaged fruits from good ones by using X-ray imaging technique [6]. X-ray photography with image analysis methods like density contours and pseudo-colors to express density changes were used as quarantine inspection of fruits such as apple, pear, 
peach, cherry tomato and orange to detect the internal injuries caused by fruit fly pest [7] and softX-ray technique aided with image processing practices such as color space conversion, adaptive threshold segmentation and Blob analysis was used to find the inside defects of pomegranate fruits [8].

Mango is the most economically important fruit of India. More than thousand mango varieties are found in India of which only 30 varieties are grown on commercial scale. India being the major producer of mangoes in the world, it is losing its shine in the world marketdue to post-harvest loss, restricted import by developed countries because of their quarantine needs and dearth of better technology for obtaining the correct information for harvesting and transportation [9]. Major reasons for post-harvest loss of mango fruits are internal injury, disease and pest attack. Some of these have no external symptoms and are not visible to naked eyes until the fruit is cut. Hence,there is need to develop a non-destructive model to separate the defective ones from good produces.

The major defects occurring in Mangoes are spongy tissue, anthracnose, seed weevil and fruit fly. Spongy tissueis a physiological disorder commonly occurring in "Alphonso" cultivar, where a desiccated sponge-like tissue is found in the flesh of ripened fruit. The reasons behind the spongy tissue may be biochemical disturbances or mechanical injury [9].Anthracnose (Colletotrichumgloesporioides) begins as hidden infections in unripe fruit and develops when the mangoes begin to ripen. Infection in larger fruits usually remains dormant until fruit ripens. Seed weevil is a major quarantine pest and is found in protected position within the seed in the center of theme so carp and near the surface. Damage is confined almost entirely to destruction of the seed which is of prime concern for quarantine regulations [10]. Fruit fly is one of the world's most destructive fruit pests which are commonly found in mango fruit. Eggs are deposited beneath the skin and its larvae feed on the flesh of the fruit resulting in damage and decay of flesh [11].

Although several studies have been reported involving different imaging techniques and computer vision on various fruits, limited work has been carried out on non-destructive quality evaluation of mangoes using X-ray imaging and neural networks. And also, there is lack of benchmark dataset availability for horticulture products like fruits. Hence in our proposed model, we build a dataset of X-ray images for mangoes and then validate the same using Discriminant Function Analysis (DFA). The X-ray images tend to be noisier than MRI or CT- scan due to the random distribution of photons within the image [12].So we develop a predictive model which determines the group membership of each sample using DFA. In the course of predicting the group membership, the features that best predicts the group membership of each image were selected. These selected features were used to train the Multilayer Perceptron Neural Network (MLPNN) to detect and classify the X-ray images as defective or non-defective.MatlabR2014 and its image processing toolbox were used for image processing. IBM SPSS Statistics 20 was used for performing discriminant analysis of samples.

\section{MATERIALS AND METHODS}

In the direction of developing a reliable, objective, nondestructive automated quality analysis technique using $\mathrm{X}$ ray imaging, the proposed model incorporates the following steps: (i) Sample collection and Image acquisition (ii) Preprocessing and segmentation (iii) Feature extraction (iv) Feature selection and Dataset validation using DFA, (v) Classification (vi) Experiments and validation.

\section{A. Sample Collection and Imge Acquisition}

The "Alphonso" mango samples were collected from a grovein Mysuru, Karnataka, a southern state of India from 2016 and 2017 harvests. The collected samples were randomly selected depending on the external appearance and cleaned from dust and dirt. X-ray images of these samples were acquired using fixed digital radiography machine from GE healthcare (used for medical purpose) at The X-ray Institute diagnostic centre, Mysuru, Karnataka. The X-ray machine consists of control panel, high tension transformer and X-ray tube head. The current $(\mathrm{mA})$, voltage $(\mathrm{kV})$ and exposure time (ms) can be set to required values in the generator control panel.The technical specifications of the $\mathrm{X}$-ray machine are given in Table I.

Table- I: Technical specifications of the X-ray machine

\begin{tabular}{|c|c|c|}
\hline \multirow{3}{*}{ X-ray Generator } & Anode voltage & $230 \mathrm{~V}$ \\
\hline & Tube Current & $0-5 \mathrm{~mA}$ \\
\hline & Penetration & $100 \mathrm{kVp}$ \\
\hline \multirow{4}{*}{$\begin{array}{l}\text { Image } \\
\text { Generating } \\
\text { System }\end{array}$} & Detector Area & $14 \times 17 \mathrm{~cm}^{2}$ \\
\hline & Image Size & 1710 X 2043 pixels \\
\hline & Image Capture & $\begin{array}{c}\text { Manual trigger } \\
\text { synchronized with the } \\
\text { X-ray generator }\end{array}$ \\
\hline & $\begin{array}{ll}\text { Image } & \text { Capture } \\
\text { Software } & \\
\end{array}$ & Image suite \\
\hline \multirow{6}{*}{ Specifications } & Power supply & $250 \mathrm{~V} \mathrm{AC}, 50 \mathrm{~Hz}$ \\
\hline & Power consumption & $2.5 \mathrm{kV}$ A maximum \\
\hline & Ambient temperature & $20-25^{\circ} \mathrm{C}$ \\
\hline & Input voltage & $400 \mathrm{~V}$ \\
\hline & Output current & $50 \mathrm{Amps}, 50 \mathrm{~Hz}$ \\
\hline & Exposure time & $0.02 \mathrm{~ms}-0.04 \mathrm{~ms}$ \\
\hline
\end{tabular}

The soft X-rays with longer wavelengths have low penetration power and are capable of revealing internal density changes [13]. Hence, based on trial and error methods, optimum output conditions of X-ray machine has been set to acquire 'soft X-ray' image of mango fruits such that internal defects are easily detectable. Accordingly, the output voltage and composite factor of the X-ray source were set to $45-50 \mathrm{kV}$ and $25 \mathrm{~mA}$ respectively, with exposure time of $0.04 \mathrm{~ms}$. At these output conditions, it was possible to get the images of mango soft tissues with better contrast revealing internal injuries and minimum noise as shown in Fig.1b.

In total 572 fruit samples were imaged (Fig. 1a). After acquiring the images, the samples were cut open manually, 
and depending on the presence or absence of defects, the $\mathrm{x}$ ray images were labeled into Defective and Non-defective (Fig. 1c). Accordingly, 277 images were labeled as defective and 295 images as non-defective.

\section{B. Pre-processing and Segmentation}

The originally acquired X-ray images were low in contrast and noisy. So, to increase the quality of the images, image enhancement technique was used followed by segmentation of fruits from their surroundings.

- Enhancement: Contrast limited adaptive histogram equalization (CLAHE) was performed to increase the contrast of the X-ray images. It first divides the entire image into smaller regions called tiles and then enhances the contrast of each of these tiles such that the histogram of each output region matches the uniform distribution of the input [14]. Since this method performs limited contrast enhancement, the noise level in the input image also reduces (Fig. 1d).

- Segmentation using thresholding: From the obtained high contrast gray value image, $I$, a binary image $J$ is obtained by marking all the pixels that have gray values greater than a certain threshold $\mathrm{T}$.

$$
J(i, j)=\left\{\begin{array}{lr}
1 & \text { if } I(i, j)>T \\
0 & \text { else }
\end{array}\right.
$$

Morphological operations were performed on the segmented image to eliminate isolated pixels in the background and to fill holes in the foreground (Fig. 1e).

\section{Feature Extraction}

The feature extraction method was applied only for gray channel as the dataset consists of X-ray image. A total of 376 geometric and intensity features were computed from segmented images of mangoes.

Geometric Features: These include standard features like location, shape information like size, area, perimeter, Euler number and solidity; Invariant moments and Fourier descriptors like shape information invariant to scale, orientation, position based on $\mathrm{Hu}$ moments [15];

Intensity Features: These include standard intensity features like standard deviation, kurtosis, skewness of the intensity in the image, boundary gradient, contrast measurements to assess the intensity difference between object and background; Haralick approach based statistical texture; Local binary patterns (LBP) based texture information based on the relationship between each pixel intensity value with its neighbors; Hu'sinvariant moments based on shape and intensityvalues[16].
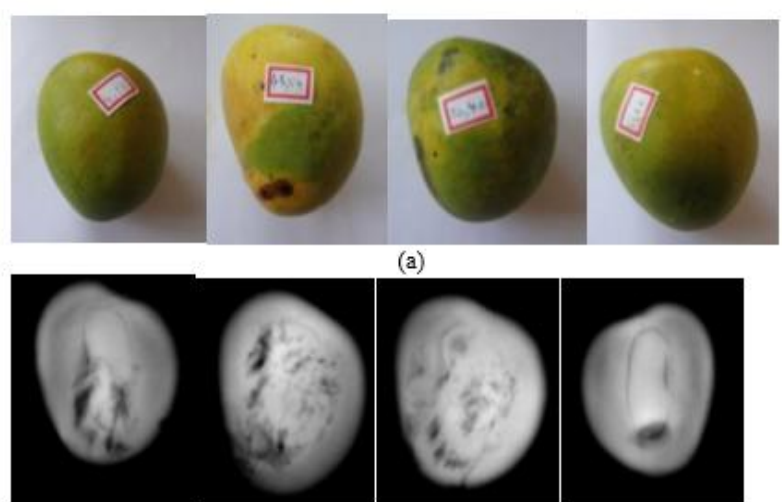

(b)

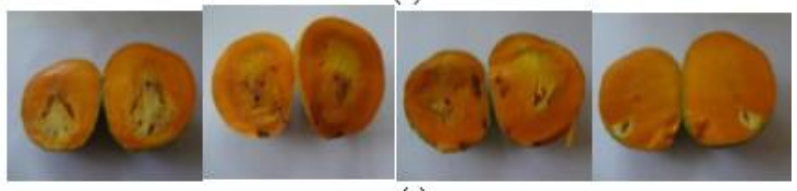

(c)

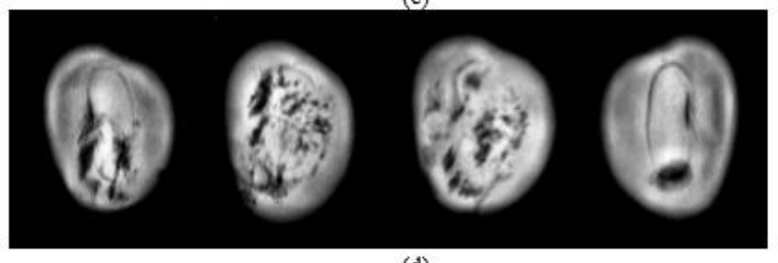

(d)

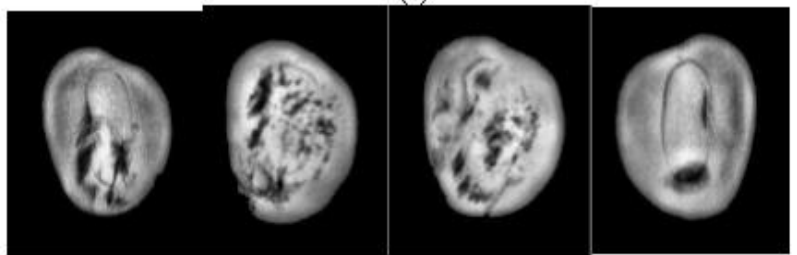

(e)

Fig. 1. Sample defective fruit images. (a) Whole fruit images (b) Original X-ray images (c) Cut open fruits (d) Enhanced images (e) Segmented images

\section{Feature Selection and Dataset Validation}

Since there is lack of benchmark dataset for mangoes $\mathrm{X}$ ray images, we have developed the dataset and built a predictive model based on discriminant function analysis which determines the group membership of each sample image, thereby validating the proposed dataset.The model consists of discriminant function based on linear combinations 376 predictor variables of both geometric and intensity features from gray channels. These features were analysed to provide the discrimination between 2 groups: defective and non-defective.

A discriminant score is calculated based on the weighted combination of the independent variables,

$$
D_{i}=a+b_{1} x_{1}+b_{2} x_{2}+\cdots+b_{n} x_{n}
$$

where ${ }^{\circ} \mathrm{D}_{\mathrm{i}}$ ' is predicted discriminant score of discriminant function, ' $\mathrm{x}$ ' is independent variable or predictor, ' $b$ ' is discriminant weight for predictor and ' $a$ ' is constant of discriminant function.

The number of discriminant functions is given by, g-1, where ' $g$ ' is the number of groups. Since the proposed model has two groups, only one discriminant function is created in the study as the linear combination of independent predictor variables that entered the model.

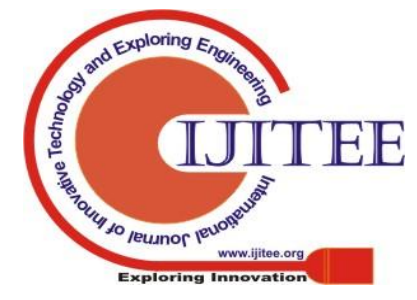


Combining Discriminant Analysis and Neural Networks for Detection of Internal Defects in Mangoes using X-Ray Imaging Technique

This function maximizes the difference between the values of the dependent variable i.e., groups. In the present study, since the total number of predictor variables is 376 , instead of entering independents together, the researcher has employed step-wise method to select the best predictor variables to be used in the model. The 31 predictor variables that entered the model and their significance in predicting the dependent are given in Table II. Among the variables that entered the model 16 are geometric features, only one from standard intensity, contrast, DCT, Hu moment and LBP feature space, 4 from Fourier, Haralick and 2 Gabor features. The predictor variables that entered the model, their standardized discriminant function coefficients, which indicate the discriminating ability and also the unstandardized discriminant function coefficients are given in Table II.

Table-II: Predictor Variables that Entered the Model

\begin{tabular}{|c|c|c|c|}
\hline \multicolumn{2}{|c|}{ Predictor Variables } & \multirow{2}{*}{$\begin{array}{l}\text { Standardized } \\
\text { Discriminant } \\
\text { Coefficients } \\
\text { of Function } 1\end{array}$} & \multirow{2}{*}{$\begin{array}{c}\text { Unstandardiz } \\
\text { ed } \\
\text { Discriminant } \\
\text { Coefficients } \\
\text { of Function } 1\end{array}$} \\
\hline Groups & Features & & \\
\hline \multirow{16}{*}{$\begin{array}{l}\text { Geometric } \\
\text { Features }\end{array}$} & center of grav i $[\mathrm{px}]$ & -.691 & -.006 \\
\hline & center of grav j [px] & -1.539 & -.016 \\
\hline & Height [px] & 5.060 & .031 \\
\hline & Width [px] & 4.151 & .032 \\
\hline & Area [px] & -1.745 & .000 \\
\hline & Perimeter [px] & -.960 & -.002 \\
\hline & Roundness & .567 & 6.795 \\
\hline & Danielsson factor & -.481 & -4.369 \\
\hline & Euler Number & .036 & .261 \\
\hline & $\begin{array}{l}\text { Equivalent Diameter } \\
{[\mathrm{px}]}\end{array}$ & -4.650 & -.033 \\
\hline & Orientation [grad] & .017 & .000 \\
\hline & Eccentricity & .195 & 4.294 \\
\hline & Hu-moment 1 & .686 & 129.417 \\
\hline & Hu-moment 2 & .124 & 167.254 \\
\hline & Hu-moment 3 & .185 & 3189.047 \\
\hline & Hu-moment 6 & .279 & 125845.293 \\
\hline $\begin{array}{l}\text { Standard } \\
\text { intensity }\end{array}$ & g-Intensity StdDev & .025 & .002 \\
\hline Contrast & g-contrast-K3 & .331 & 3.413 \\
\hline \multirow[t]{4}{*}{ Haralick } & g-Tx 3,d1(mean ) & -.726 & -9.476 \\
\hline & g-Tx 6,d1(range) & .825 & 34.733 \\
\hline & g-Tx 1,d2(mean ) & .069 & 1.500 \\
\hline & g-Tx 1,d3(range) & -.815 & -14.748 \\
\hline \multirow{5}{*}{$\begin{array}{l}\text { Fourier } \\
\text { and DCT }\end{array}$} & g-Fourier Abs $(2,4)$ & -.323 & -.001 \\
\hline & g-Fourier Abs $(3,3)$ & -.194 & -.001 \\
\hline & $\begin{array}{l}\text { g-Fourier Ang } \\
(2,2)[\mathrm{rad}]\end{array}$ & .116 & .670 \\
\hline & $\begin{array}{l}\text { g-Fourier Ang } \\
(3,4)[\mathrm{rad}]\end{array}$ & -.081 & -.510 \\
\hline & g-DCT $(1,4)$ & -.429 & -.019 \\
\hline $\begin{array}{l}\text { Hu with } \\
\text { intensity }\end{array}$ & g-Hu-moment-int 4 & -.165 & -155.478 \\
\hline
\end{tabular}

\begin{tabular}{|l|l|l|l|}
\hline \multicolumn{2}{|c|}{ Predictor Variables } & \multirow{2}{*}{$\begin{array}{c}\text { Standardized } \\
\text { Discriminant } \\
\text { Coefficients } \\
\text { of Function 1 }\end{array}$} & $\begin{array}{c}\text { Unstandardiz } \\
\text { ed } \\
\text { Discriminant } \\
\text { Coefficients } \\
\text { of Function 1 }\end{array}$ \\
\hline Groups & \multicolumn{1}{|c|}{ Features } & .448 & .237 \\
\hline LBP & $\mathrm{g}-$ Gabor(2,6) & .520 & .264 \\
\cline { 2 - 4 } & $\mathrm{g}-$ g-LBbor(7,6) & .440 & .000 \\
\hline (constant) & & - & -46.954 \\
\hline
\end{tabular}

The larger the absolute values of standardized discriminant functioncoefficients, greater the corresponding variable's discriminating ability. So from the table we observe that some geometric features such as height, width, roundness and selected intensity features like contrast, Haralick, Gabor and LBP have significant discriminating ability.

The discriminant function of the modelbuilt from the unstandardized discriminant coefficients is:

$\mathrm{D}_{\mathrm{i}}=-46.954-0.006$ center of grav $\mathrm{i}-0.016$ center of grav $\mathrm{j}$ +0.031 Height $+\ldots \ldots \ldots \ldots+0.000$ g-LBP $(1,15)[8, \mathrm{u} 2]$

Since our model has two groups, the centroids are calculated based on above mentioned discriminant function. TableIII is used to establish the cutting point for classifying cases. With two unequal groups, the optimal cutting point will be the weighted average of the two values.

Table III: Functions at Group Centroids

\begin{tabular}{|l|l|}
\hline \multicolumn{1}{|c|}{ Group } & \multicolumn{1}{c|}{ Function 1 } \\
\hline Defective & -1.148 \\
\hline Non-defective & 1.078 \\
\hline
\end{tabular}

The plots of canonical discriminant function for two groups, defective and non-defective are given in Fig. 2. Since the two distributions do not overlap too much we can conclude that the discriminant function is good and discriminates the groups significantly. 


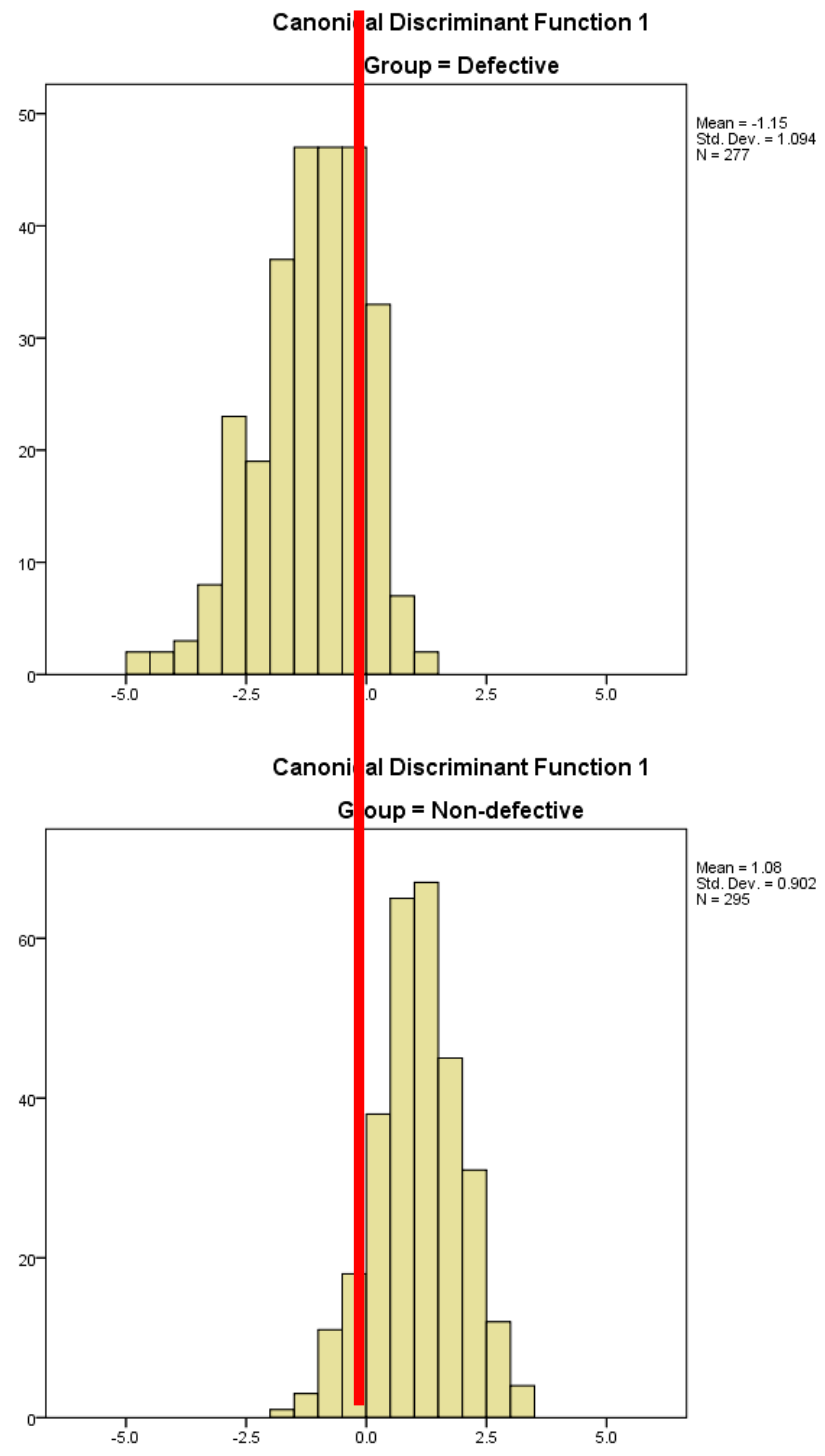

Fig. 2. Canonical Function Plots of Defective and Nondefective Groups

\section{E. Disease Classification}

With optimized dataset and selected features, MLP NN predictive model was used to classify mangoes into two classes. MLP is a feed forward, fully connected, supervised learning neural network with one or two hidden layers. Neurons of each hidden layer are a function of weighted sum of their inputs which are passed to the neurons of subsequent layer through non-linear activation functions. In this study, the performance of MLP topology with one and two hidden layers was examined.Various non-linear activation functions were used for the hidden layers and output layer to determine the combination of activation functions yielding maximum classification accuracy. The hidden layer activation functions were set to hyperbolic tangent and sigmoid, whereas identity, softmax, hyperbolic tangent and sigmoid activation functions were used for output layer. The network learning is achieved through back-propagation algorithm. Scaled conjugate gradient (SCG)optimization algorithm was considered for minimizing the training error by updating the synaptic weights of each neuron. Performance of this algorithm was evaluated for different MLP architectures.

The labeled dataset was partitioned into train and test samples in the ratio of 80:20. To avoid overtraining, the test sample was built as an independent set from that of train sample. The 31 predictor variables, i.e., features that entered the predictive model were rescaled by mean subtraction and dividing by standard deviation. These features form the first layer and the two categorical outputs, defective and nondefective, form the last layer of the network topology. Since the proposed dataset is much smaller in size, batch type training was performed where the weights were updated only after passing all training samples thereby directly minimizing the total error.

The scaled conjugate gradient algorithm is a variation oflinear search based conjugate gradient algorithmsand it is the combination of model-trust region based LevenbergMarquardt algorithm and conjugate gradient approach [17]. Unlike other conjugate gradient algorithms where the performance is based on user fed parameters, SCG does not depend on any of those and is also faster than latter as it uses stepwise optimization instead of time consuming line search for every iteration.

The following training options were set: Initial $\lambda$ $($ lambda $)=0.0000005$ and initial $\sigma($ sigma $)=0.00005$. In the proposed model different MLP architectures were created with one and two hidden layers and different activation functions at the hidden and output layer. The activation function of hidden layer/layers was set to hyperbolic tangent and sigmoid and that of output layer was checked for identity, soft max, hyperbolic tangent and sigmoid. Cross entropy error function was used for softmax function and sum-of-square for the remaining activation functions. The model was tested with different number of neurons in the hidden layer/layers to get an optimized classification results.

\section{RESULTS AND DISCUSSIONS}

A huge feature space, consisting of 376 features, was built by extracting size and textural features from each image of the proposed dataset and then discriminant analysis was performed to obtain the a set of 31 features that best predicts the group membership of each image into two classes. In this way, it was possible to extract and select relevant features in an off-line process, thereby decreasing the computational cost during on-line disease classification process. Then, these selected features were used as predictor variables for MLP NN classifier with SCG optimization algorithm to achieve high classification performance. The train and test accuracy of different MLP architectures in terms of train and test accuracy is summarized in Table IV. 
Combining Discriminant Analysis and Neural Networks for Detection of Internal Defects in Mangoes using X-Ray Imaging Technique

Table IV: Performance Summary of Different MLP Architectures

\begin{tabular}{|c|c|c|c|c|c|}
\hline \multirow{2}{*}{$\begin{array}{c}\text { Activation } \\
\text { Function of } \\
\text { Hidden } \\
\text { Layer/Layers }\end{array}$} & \multirow{2}{*}{$\begin{array}{c}\text { Activation } \\
\text { Function of } \\
\text { Output Layer }\end{array}$} & \multicolumn{2}{|c|}{$\begin{array}{c}\text { Accuracy for } \\
\text { One Hidden } \\
\text { Layer (in \%) }\end{array}$} & \multicolumn{2}{|c|}{$\begin{array}{c}\text { Accuracy for } \\
\text { Two Hidden } \\
\text { Layers (in } \\
\% \text { ) }\end{array}$} \\
\hline & & Train & Test & Train & Te \\
\hline \multirow{4}{*}{ Sigmoid } & Identity & 89 & 84 & 86.7 & $\begin{array}{c}87 . \\
5\end{array}$ \\
\hline & Softmax & 86.5 & 88.5 & 87.8 & $\begin{array}{c}84 . \\
1\end{array}$ \\
\hline & $\begin{array}{l}\text { Hyperbolic } \\
\text { Tangent }\end{array}$ & 90.4 & 83.6 & 86.5 & $\begin{array}{c}87 . \\
4\end{array}$ \\
\hline & Sigmoid & 92.3 & 89.1 & 88.7 & $\begin{array}{c}84 . \\
4\end{array}$ \\
\hline \multirow{4}{*}{$\begin{array}{l}\text { Hyperbolic } \\
\text { Tangent }\end{array}$} & Identity & 88.4 & 86.2 & 89.9 & $\begin{array}{c}86 . \\
7\end{array}$ \\
\hline & Softmax & 92 & 89.3 & 82.5 & 89 \\
\hline & $\begin{array}{l}\text { Hyperbolic } \\
\text { Tangent }\end{array}$ & 90.7 & 86.6 & 92.6 & 83 \\
\hline & Sigmoid & 87.7 & 91.3 & 87 & $\begin{array}{c}89 . \\
2\end{array}$ \\
\hline
\end{tabular}

We see from the above table that the best MLP architecture is the one with train accuracy of $87.7 \%$ and test accuracy of $91.3 \%$. It is built with one hidden layer, hidden layer activation function of hyperbolic tangent and output layer activation function of sigmoid with sum-of-squares error function. It is also observed that as we increased the number of hidden layers to two, the classification accuracy for test sample was much lower than that of one hidden layer.

Fig. 3 shows the optimized MLP topology with one hidden layer, 31 predictor values at the input layer, 10 neurons at the hidden layer/layers and two categorical classes at the output layer. The network performance for the above mentioned optimized architecture based on the combination of train and test samples are given in Table $\mathrm{V}$ as model summary.

Table V: Model summary

\begin{tabular}{|c|c|c|}
\hline \multirow{4}{*}{ Training } & Sum of Squares Error & 43.676 \\
\hline & Incorrect Predictions & $12.3 \%$ \\
\hline & Stopping Rule Used & $\begin{array}{l}1 \text { consecutive step(s) with no } \\
\text { decrease in error }\end{array}$ \\
\hline & Training Time & 0:00:00.20 \\
\hline \multirow{2}{*}{ Testing } & Sum of Squares Error & 8.798 \\
\hline & Incorrect Predictions & $8.7 \%$ \\
\hline
\end{tabular}

From the model summary we observe that, the error is the sum-of-squares as the activation function at the output layer is sigmoid, and since the output layer is categorical in nature, the relative error is given in terms of average percentage of incorrect predictions. It is $12.3 \%$ for training set and $8.7 \%$ for testing test.

The training time is 00.20 seconds. The training of model will stop when there is minimum or no decrease in error between two successive steps.

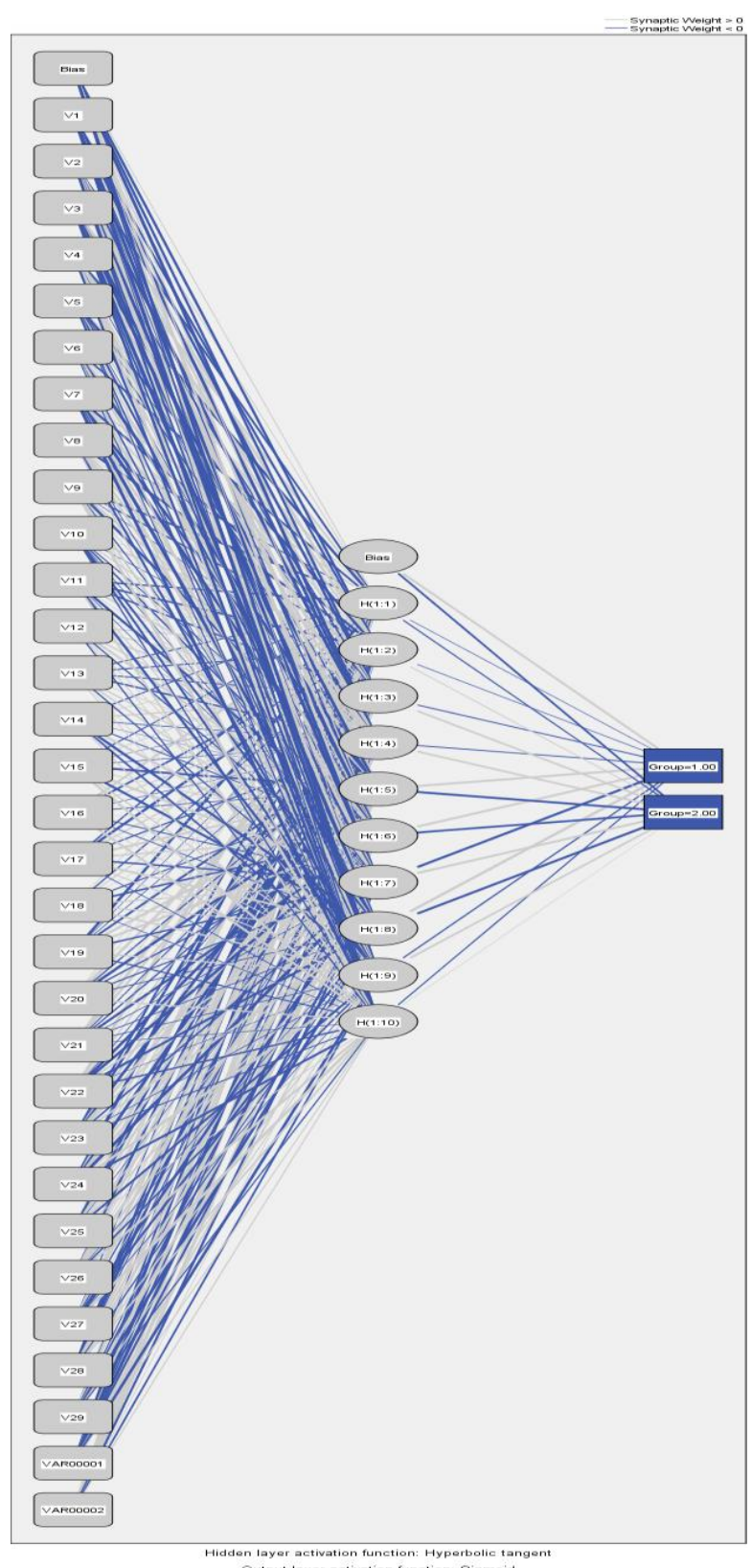

Fig. 3. The MLP NN topology of the study with one hidden layer

Table VI displays the classification summary of each class by partition and overall. The overall percentage of correct predictions during training is $87.7 \%$ and $91.3 \%$ for test samples.

Table VI: Classification Summary

\begin{tabular}{|c|l|c|c|c|}
\hline \multirow{3}{*}{ Sample } & \multirow{2}{*}{ Observed } & \multicolumn{3}{|c|}{ Predicted } \\
\cline { 3 - 5 } & Defective & Non-Defective & $\begin{array}{c}\% \\
\text { correct }\end{array}$ \\
\hline \multirow{3}{*}{ Training } & Defective & 194 & 24 & $89 \%$ \\
\cline { 2 - 5 } & Non-Defective & 32 & 207 & $86.6 \%$ \\
\cline { 2 - 5 } & Overall Percent & $49.5 \%$ & $50.5 \%$ & $87.7 \%$ \\
\hline \multirow{3}{*}{ Testing } & Defective & 52 & 7 & $88.1 \%$ \\
\cline { 2 - 5 } & Non-Defective & 3 & 53 & $94.6 \%$ \\
\hline
\end{tabular}


Overall Percent

$47.8 \%$

$52.2 \%$

$91.3 \%$

\section{CONClusions}

In this work, we have proposed a predictive model for internal quality analysis of Mango fruits by combining the discriminant function analysis and MLP NN on X-ray imaging technology. The two non-overlapping regions of DFA plot indicates that the samples in the manually prepared X-ray dataset are distinct and belong to two groups, defective and non-defective, with less discrimination among samples of the same group. During the process of DFA, the 31 input features that entered the predictive and that best discriminates the samples among two groups are used to train the MLP NN classifier. Different architectures of MLP were tested by varying the number of hidden layers from one to two and using various non-linear activation functions at the hidden and output layer. We observed that, in MLP NN the accuracy of test dataset decreases with more number of hidden layers as the network overfits to the training dataset. The network stops learning the trend and thus was not able to generalize to new data samples in test dataset resulting in lower classification accuracy. The use of SCG optimization algorithm in MLP further reduced the burden of handpicking the network parameters. Thus, in this study, we built a real time quality analysis system where extraction and selection of features was done in off-line and on-line disease classification with accuracy of $91.3 \%$ was done at decreased computational cost and elapsed time, the training time of the proposed model was 00.20 seconds.

\section{REFERENCES}

1. Barcelon, Eufemio G., Seishu Tojo, and Kengo Watanabe. "Relating X-ray absorption and some quality characteristics of mango fruit." Journal of agricultural and food chemistry 47, no. 9: 38223825, 1999.

2. Ruiz-Altisent, Margarita, Luis Ruiz-Garcia, G. P. Moreda, Renfu Lu, Natalia Hernandez-Sanchez, E. C. Correa, B. Diezma, B. Nicolaï, and J. García-Ramos. "Sensors for product characterization and quality of specialty crops-A review." Computers and Electronics in agriculture 74, no. 2: 176-194, 2010.

3. Veena, T., D. V. Chidanand, and K. Alagusundaram. "Quality analysis of mango fruit with fruit fly insect by non-destructive soft Xray method." International Journal of Agricultural Science and Research (IJASR) 5, no. 3: 37-45, 2015.

4. Kotwaliwale, Nachiket, Karan Singh, Abhimannyu Kalne, Shyam Narayan Jha, Neeraj Seth, and Abhijit Kar. "X-ray imaging methods for internal quality evaluation of agricultural produce." Journal of food science and technology 51, no. 1: 1-15, 2014.

5. Kim, S., and T. F. Schatzki. "Apple watercore sorting system using X-ray imagery: I. Algorithm development." Transactions of the ASAE 43, no. $6: 1695,2000$.

6. Chen, Pictiaw, and Z. Sun. "A review of non-destructive methods for quality evaluation and sorting of agricultural products." Journal of Agricultural Engineering Research 49: 85-98, 1991.

7. "Non-Destructive Quarantine Technique? Potential Application of Using X-Ray Images to Detect Early Infestations Caused by Oriental Fruit Fly (Bactrocera dorsalis)(Diptera: Tephritidae) in Fruit." Formosan Entomologist 26, no. 2: 171-186, 2006.

8. Payel, G., and C. K. Sunil. "Quality Analysis of Pomegranate by Non-Destructive Soft X-Ray Method." J Food Process Technol 5, no. 341: 2, 2014.

9. Jha, S. N., K. Narsaiah, A. D. Sharma, Manpreet Singh, Sunil Bansal, and R. Kumar. "Quality parameters of mango and potential of nondestructive techniques for their measurement-a review." Journal of food science and technology 47, no. 1: 1-14, 2010.

10. Ullagaddi, Shivayogi B., and Vishwanadha Raju." International journal of graphics and multimedia (IJGM)." Journal Impact Factor 5, no. $1: 36-45,2014$
11. Kader, Adel A., and Rosa Sonya Rolle. The role of post-harvest management in assuring the quality and safety of horticultural produce. Vol. 152. Food \& Agriculture Org., 2004.

12. Sprawls, Perry. Physical principles of medical imaging. Aspen Publishers, 1987.

13. Chuang, Cheng-Long, Cheng-Shiou Ouyang, Ta-Te Lin, Man-Miao Yang, En-Cheng Yang, Tze-Wei Huang, Chia-Feng Kuei, Angela Luke, and Joe-Air Jiang. "Automatic X-ray quarantine scanner and pest infestation detector for agricultural products." Computers and Electronics in Agriculture 77, no. 1: 41-59, 2011.

14. Pizer, Stephen M., R. Eugene Johnston, James P. Ericksen, Bonnie C. Yankaskas, and Keith E. Muller. "Contrast-limited adaptive histogram equalization: speed and effectiveness." In [1990] Proceedings of the First Conference on Visualization in Biomedical Computing, pp. 337-345. IEEE, 1990.

15. Mery, Domingo, Franco Pedreschi, and Alvaro Soto. "Automated design of a computer vision system for visual food quality evaluation." Food and Bioprocess Technology 6.8, 2093-2108, 2013.

16. Mery, Domingo and Alvaro Soto. "Features: the more the better." Proceedings of the 8th conference on Signal processing, computational geometry and artificial vision, World Scientific and Engineering Academy and Society (WSEAS), Rhodes, Greece, 2008.

17. Møller, Martin Fodslette. "A scaled conjugate gradient algorithm for fast supervised learning." Neural networks 6, no. 4: 525-533, 1993.

\section{AUTHOR PROFILE}

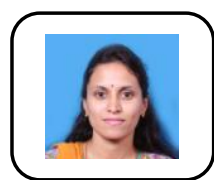

Vani Ashok obtained her Masters degree in Computer Engineering in the year 2003. She is pursuing research in the area of image processing and artificial intelligence. She is working a Assistant Professor in the Department of Computer Science and Engineering at Sri Jayachamarajendra College of Engineering, Mysuru, Karnataka, India. She has presented papers in international conferences and published paper in international journal. 